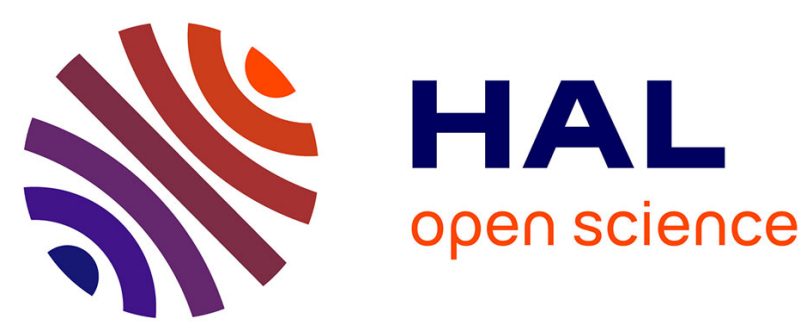

\title{
Bacterial Identification Using 16S rDNA Gene Sequencing and Antibiogram Analysis on Biofield Treated Pseudomonas fluorescens
}

Mahendra Kumar Trivedi, Alice Branton, Dahryn Trivedi, Gopal Nayak, Mayank Gangwar, Snehasis Jana

\section{To cite this version:}

Mahendra Kumar Trivedi, Alice Branton, Dahryn Trivedi, Gopal Nayak, Mayank Gangwar, et al.. Bacterial Identification Using 16S rDNA Gene Sequencing and Antibiogram Analysis on Biofield Treated Pseudomonas fluorescens. Clinical \& Medical Biochemistry: Open Access, 2015, 1 (1), pp.1000101. hal-01416081

\section{HAL Id: hal-01416081 \\ https://hal.science/hal-01416081}

Submitted on 14 Dec 2016

HAL is a multi-disciplinary open access archive for the deposit and dissemination of scientific research documents, whether they are published or not. The documents may come from teaching and research institutions in France or abroad, or from public or private research centers.
L'archive ouverte pluridisciplinaire HAL, est destinée au dépôt et à la diffusion de documents scientifiques de niveau recherche, publiés ou non, émanant des établissements d'enseignement et de recherche français ou étrangers, des laboratoires publics ou privés. 


\title{
Bacterial Identification Using 16S rDNA Gene Sequencing and Antibiogram Analysis on Biofield Treated Pseudomonas fluorescens
}

Mahendra Kumar Trivedi', Alice Branton'1, Dahryn Trivedi', Gopal Nayak', Mayank Gangwar ${ }^{2}$ and Snehasis Jana $^{{ }^{*}}$

${ }^{1}$ Trivedi Global Inc., 10624 S Eastern Avenue Suite A-969, Henderson, NV 89052, USA

${ }^{2}$ Trivedi Science Research Laboratory Pvt. Ltd., Hall-A, Chinar Mega Mall, Chinar Fortune City, Hoshangabad Rd., Bhopal, Madhya Pradesh, India

\begin{abstract}
Biofield therapies have been reported to improve the quality of life as compared to other energy medicine. The aim of the study was to evaluate the impact of Mr. Trivedi's biofield energy treatment on Pseudomonas fluorescens $(P$. fluorescens) for antimicrobial sensitivity, minimum inhibitory concentration (MIC), biochemical reactions, and biotype number. $P$. fluorescens cells were procured from MicroBioLogics Inc., USA in sealed packs bearing the American Type Culture Collection (ATCC 49838) number and divided in control and treated group. The effect was evaluated on day 10, and 159 after biofield treatment in lyophilized state. Further study was performed on day 5, 10, and 15 after retreatment on day 159 in revived state as per study design. All experimental parameters were studied using automated MicroScan Walk-Away ${ }^{\circledR}$ system. The $16 \mathrm{~S}$ rDNA sequencing was carried out to correlate the phylogenetic relationship of $P$. fluorescens with other bacterial species after treatment. The results showed improved sensitivities and decreased MIC value of aztreonam, cefepime, moxifloxacin, and tetracycline in revived and lyophilized treated sample with respect to the control. Arginine, cetrimide, kanamycin, and glucose showed altered biochemical reactions after biofield treatment with respect to control. Biotype numbers were altered along with species in lyophilized as well as in revived group. Based on nucleotides homology and phylogenetic analysis using 16S rDNA gene sequencing, treated sample was detected to be Pseudomonas entomophila (GenBank Accession Number: AY907566) with $96 \%$ identity of gene sequencing data, which was nearest homolog species to $P$. fluorescens (Accession No. EF672049). These findings suggest that Mr. Trivedi's unique biofield treatment has the capability to alter changes in pathogenic $P$. fluorescens even in the lyophilized storage condition and can be used to modify the sensitivity of microbes against antimicrobials.
\end{abstract}

Keywords: Pseudomonas fluorescens; Biofield treatment; Lyophilized state; Antimicrobial susceptibility; Biochemical reactions; Biotype; $16 \mathrm{~S}$ rDNA sequencing

\section{Abbreviations \\ NCCAM: National Center for Complementary and Alternative Medicine; ATCC: American Type Culture Collection; DNA: Deoxyribonucleic acid; ESBL: Extended spectrum beta-lactamase; MIC: Minimum inhibitory concentration; MEGA: Molecular evolutionary genetics analysis; NBPC30: Negative breakpoint combo panel 30; NCBI: National center for biotechnology information; OTUs: Operational Taxonomic Units; PCR: Polymerase chain reaction; RDP: Ribosomal database project}

\section{Introduction}

The genus Pseudomonas is a group of ubiquitous Gram-negative rod shaped bacterium mostly present in soil, and water surfaces. Pseudomonas infections are related with high morbidity and mortality [1], and common species isolated from clinical specimen is Pseudomonas aeruginosa [2]. However, Pseudomonas fluorescens ( $P$. fluorescens) is a member of the fluorescent pseudomonas group and mostly regarded to be of low virulence and an infrequent human infection [3]. P. fluorescens is a heterogeneous species that can be subdivided by various taxonomic criteria into several biotypes [4]. Complete genome sequence was reported in two strains, namely $P$. fluorescens Pf-5 genome consist of 87 RNAs and 6137 proteins, and $P$. fluorescens $\mathrm{PfO}-1$ genome consist of 95 RNAs and 5736 proteins $[5,6]$.

Lyophilization or freeze drying is the most widely used storage methods for drying bacteria or other biological materials. It may be considered as a two-stage process of freezing and drying. Freezing has been extensively used in case of cells and tissues [7], which can be convenient for the survival of microorganisms. Even sensitive microorganism showed satisfactory recoveries, if proper care must be taken with respect to temperature control, suspending media, and rate of cooling [8]. On the other hand, drying is also more advisable storage condition for sensitive microorganism. Above methods involved removal of moisture from a frozen solution or suspension in a high vacuum, but few exceptions in case of nonviable materials, especially proteins and tissues. This approach has been extensively used for different substance in past 40 years by numerous investigators $[9,10]$. Although, alterations in microbes cannot be happened in lyophilized state without any strong energy transmission. In order to evaluate the impact of Mr. Trivedi's biofield treatment on lyophilized strain, study was designed to investigate the alteration in antibiogram pattern and its related parameters. Mr. Trivedi has the ability to harness the energy from the environment or Universe and transmit this energy into any object (living or nonliving) on the Globe. The objects always receive the energy and responding into useful way that is knows biofield energy. This process is termed as biofield treatment. Mr. Trivedi's unique biofield treatment is also known as The Trivedi Effect. Biofield, the electromagnetic field that surrounds the living organism will provides regulatory and communication functions within the organism. Mr. Trivedi's biofield energy treatment was extensively studied in material

*Corresponding author: Dr. Snehasis Jana, Trivedi Science Research Laboratory Pvt. Ltd., Hall-A, Chinar, Mega Mall, Chinar Fortune City, Hoshangabad Rd., Bhopal-462 026, Madhya Pradesh, India, Tel: +91-755-6660006; E-mail: publication@trivedisrl.com

Received August 27, 2015; Accepted August 31, 2015; Published September 04, 2015

Citation: Trivedi MK, Branton A, Trivedi D, Nayak G, Gangwar M, et al. (2015) Bacterial Identification Using 165 rDNA Gene Sequencing and Antibiogram Analysis on Biofield Treated Pseudomonas fluorescens. Clin Med Biochemistry Open Access 1: 101. doi:10.4172/2471-2663.1000101

Copyright: @ 2015 Trivedi MK, et al. This is an open-access article distributed under the terms of the Creative Commons Attribution License, which permits unrestricted use, distribution, and reproduction in any medium, provided the original author and source are credited. 
Citation: Trivedi MK, Branton A, Trivedi D, Nayak G, Gangwar M, et al. (2015) Bacterial Identification Using 16S rDNA Gene Sequencing and Antibiogram Analysis on Biofield Treated Pseudomonas fluorescens. Clin Med Biochemistry Open Access 1: 101. doi:10.4172/24712663.1000101

Page 2 of 9

science [11-13], agricultural science [14-16], in biotechnology [17]. It is reported in altering the susceptibility of antimicrobials of pathogenic microbes [18-20] and reduced the viral load in hepatitis $C$ virus [21].

After consideration of clinical significance of $P$. fluorescens and significant impact of biofield treatment on microbes, a detailed investigation was studied to evaluate the impact of biofield treatment on $P$. fluorescens. Treatment was assessed in relation to antimicrobials susceptibility, biotyping based on various biochemical reactions. Further, genotyping of this organism was performed using $16 \mathrm{~S}$ rDNA sequencing method.

\section{Materials and Methods}

Lyophilized sample of Pseudomonas fluorescens [American Type Culture Collection (ATCC) 49838] was procured from MicroBioLogics, Inc., USA, and stored as per suggested storage conditions until further use. The acceptability of the identification media and antimicrobial agents were checked prior to the study by ATCC organisms. The antimicrobial susceptibility, biochemical reactions, and biotype number were evaluated on MicroScan Walk-Away" (Dade Behring Inc., West Sacramento, CA) using Negative Breakpoint Combo 30 (NBPC30) panel. The NBPC30 panel was stored at 2 to $-25^{\circ} \mathrm{C}$. The panels were allowed to equilibrate to room temperature prior to rehydration. All opened panels were used in same day. The $16 \mathrm{~S}$ rDNA sequencing study was carried out using Ultrapure Genomic DNA Prep Kit; Cat KT 83 (Bangalore Genei, India).

\section{Inoculum preparation}

The turbidity standard technique using direct inoculation of revived and lyophilized $P$. fluorescens was used. Using a sterile wooden applicator stick or bacteriological loop, the surface of 4-5 large or 5-10 small morphologically similar culture was touched for well-isolated colonies from an 18-24 hour non-inhibitory agar plate. Further, colonies were emulsified in $3 \mathrm{~mL}$ of inoculum water (autoclaved deionized water) to an equivalent of a $0.5 \mathrm{McF}$ arland barium sulfate turbidity standard. $100 \mu \mathrm{L}$ of the standardized suspension was pipetted into $25 \mathrm{~mL}$ of inoculum water using pluronic and inverted 8-10 times.

\section{Experimental design}

Experimental design for lyophilized sample of $P$. fluorescens was divided into two main groups (Gr.) namely- Gr I and Gr. II

Group I: No treatment was given. Revived from lyophilized state and considered as control. It was analyzed for antimicrobial sensitivity, minimum inhibitory concentration (MIC), biochemical reactions and biotype number as per the standard protocol.

Group II: This group was divided into two separate sub parts named as Gr. IIA and Gr. IIB.

Group IIA: P. fluorescens sample was subjected to the Mr. Trivedi's biofield treatment for the first time in the lyophilized state itself and then revived. After treatment, the analysis was done on day 10 for antimicrobial sensitivity, MIC, biochemical reactions and biotype number as per the standard protocols. Further, $16 \mathrm{~S}$ rDNA sequencing study was carried out in treated sample to study the genotypic alteration in organism.

Group IIB: It included the sample which was analyzed in Gr. IIA. P. fluorescens strain was stored for 159 days at $-70^{\circ} \mathrm{C}$, so that no contamination would take place till they were revived again. Gr. IIB was further subdivided in two groups named as Gr. IIB, study I and Gr. IIB, study II.
Group IIB-Study I: After 159 days, antimicrobial sensitivity, MIC, biochemical reactions and biotyping were performed as per the standard protocol.

Group IIB-Study II: The stored strain was revived from $-70^{\circ} \mathrm{C}$ and the revived culture was again subjected to Mr. Trivedi's biofield energy treatment (re-treatment) on day 159. After biofield retreatment, the sample was sub-cultured into three separate tubes on 3 different days (Day 0, Day 5 and Day 10) and was analyzed keeping the main treated tube aside. Each sample was analyzed after 5 days of its sub-culturing.

\section{Biofield treatment modalities}

The lyophilized (Gr. IIA) sample of $P$. fluorescens was subjected to biofield treatment followed by retreatment after storing for 159 days in revived state (Gr. IIB, study II). The treatment group in sealed pack was handed over to Mr. Trivedi for biofield treatment under laboratory condition. Mr. Trivedi provided the treatment through his energy transmission process to the treated groups without touching the samples. Treated samples were assessed for antimicrobial sensitivity, biochemical reactions, and biotyping as per experimental design. Whilst handing over these cultures to Mr. Trivedi for retreatment purposes, optimum precautions were taken to avoid contamination. The 16S rDNA gene sequencing of $P$. fluorescens was also carried out to confirm the identity of sample after biofield treatment [19].

\section{Antimicrobial sensitivity assay}

Antimicrobial sensitivity pattern of $P$. fluorescens in each group was carried out with the help of automated instrument, MicroScan WalkAway using NBPC30 panel, as per the manufacturer's instructions [22]. MIC and the qualitative susceptibility pattern like resistant (R), intermediate (I), or susceptible (S), were determined by observing the lowest antimicrobial concentration showing growth inhibition. All these antimicrobials used in this study were purchased from SigmaAldrich, USA.

\section{Biochemical studies}

The biochemical studies of $P$. fluorescens were performed on MicroScan Walk-Away [22]. Biochemical reactions pattern were carried out in all the tested group using 33 biochemicals viz. acetamide, adonitol, arabinose, arginine, cetrimide, cephalothin, citrate, colistin, esculin hydrolysis, nitrofurantoin, glucose, hydrogen sulfide, indole, inositol, kanamycin, lysine, malonate, melibiose, nitrate, oxidationfermentation/glucose, galactosidase, ornithine, oxidase, penicillin, raffinose, rhamnose, sorbitol, sucrose, tartrate, tryptophan deaminase, tobramycin, urea, and Voges-Proskauer. All these biochemicals used in this experiment were procured from Sigma-Aldrich, USA.

\section{Biotype number}

The biotype number of $P$. fluorescens was determined by automated MicroScan Walk-Away processed panel data utilizing biochemical reactions [22].

\section{PCR amplification and gene sequencing of 16S rDNA}

Genomic DNA was isolated and purified from treated group of $P$. fluorescens cells by using genomic purification Kit, as per the manufacturer's instructions. The PCR product was bi-directionally sequenced using the forward, reverse and an internal primer. DNA $16 \mathrm{~S}$ region amplification was performed using the primer set $16 \mathrm{SF}-$ 16SR [23]. The 16S rDNA gene $(\sim 1.5 \mathrm{~kb})$ was amplified employing universal primers (16SF 5' -AGAGTTTGATCCTGGCTCAG-3'; 16SR 5'-CTACGGCTACCTTGTTACGA-3'). Amplification was carried out 
Citation: Trivedi MK, Branton A, Trivedi D, Nayak G, Gangwar M, et al. (2015) Bacterial Identification Using 16S rDNA Gene Sequencing and Antibiogram Analysis on Biofield Treated Pseudomonas fluorescens. Clin Med Biochemistry Open Access 1: 101. doi:10.4172/24712663.1000101

Page 3 of 9

in a Rapid Cycler thermo controller, with initial denaturation, annealing and extension temperature. Following amplification, products were analyzed by gel electrophoresis at $100 \mathrm{~V}$ (in $1.0 \%$ agarose gel, $0.2 \mu \mathrm{g}$ of ethidium bromide $\mathrm{mL}^{-1}$ ) in tris-acetate buffer (TAE), and visualized under UV light in a gel documentation unit (BioRad Laboratories, USA). The amplified fragment of PCR was purified from the agarose gel by DNA Gel Extraction Kit. Sequencing of amplified product was carried out on commercial basis from Bangalore Genei, India. The obtained $16 \mathrm{~S}$ rDNA sequences data were aligned and compared with the sequences, available in GenBank database of National Center for Biotechnology Information (NCBI) using the algorithm BLASTn program. The multiple sequence alignment/phylogenetic tree were constructed using MEGA 3.1 software using neighbor joining method [24].

\section{Results}

\section{Evaluation of antimicrobial susceptibility}

The results of antimicrobial sensitivity after biofield treatment on P. fluorescens are summarized in Table 1. Biofield treated cells of $P$. fluorescens showed alteration in sensitivity pattern of aztreonam, cefepime, Cefotaxime, ciprofloxacin, Piperacillin, and tetracycline. Biofield treatment in lyophilized state of $P$. fluorescens showed improved sensitivity in case of tetracycline i.e., from intermediate to susceptible in Gr. IIA, on day 10 as compared to its control. Rest of the tested antimicrobials did not show any alteration in Gr. IIA, on day 10 with respect of control.

All the antimicrobials were again tested on Gr. IIB, study I sample and results showed alteration in two antimicrobials i.e., Cefepime and Cefotaxime. Cefepime showed improved sensitivity i.e., from intermediate to susceptible, while sensitivity of Cefotaxime was changed from intermediate to resistant (Gr. IIB, study I) as compared to control (Gr. I). only tetracycline showed changed sensitivity (i.e., $S \rightarrow I$ ) in $G r$. IIB, study I (day 159) as compared to analysis done on day 10 (Gr. IIA). However, results showed that most of the tested antimicrobials (except cefepime and Cefotaxime) showed sustained sensitivity profile on storage of treated $P$. fluorescens strain for 159 days as compared to control.

Further study II (Gr. IIB) results showed alteration in sensitivity pattern of tested antimicrobials after biofield retreatment in revived state of $P$. fluorescens, and compared with control. Results showed that sensitivity was improved in case of aztreonam and Cefotaxime antibiotics i.e., from resistant to intermediate on day 10 (Gr. IIB, study II) after retreatment compared with Gr. IIB, study I. Cefotaxime showed altered sensitivity pattern from intermediate to resistant on day 5 and 15 (Gr. IIB, study II) as compared to control. Ciprofloxacin showed altered sensitivity from susceptible to intermediate on day 15 (Gr. IIB, study II) after retreatment as compared to control. Piperacillin sensitivity was also altered from susceptible to intermediate on day 5 and day 15 (Gr. IIB, study II) with respect to control. Tetracycline sensitivity was improved i.e., from intermediate to susceptible on day 10 (Gr. IIB, study II) as compared to control (Gr. I). Rest of antimicrobials such as Amikacin, Ceftazidime, ceftriaxone, chloramphenicol, gentamicin, Imipenem, levofloxacin, Meropenem, Piperacillin/ tazobactam, Ticarcillin/k-clavulanate, tobramycin, and trimethoprim/ sulfamethoxazole did not show any alteration after treatment in lyophilized or revived state with respect to control.

\section{Determination of minimum inhibitory concentration (MIC)}

Biofield treatment in lyophilized and retreatment in revived $P$. fluorescens showed alteration in MIC values of antimicrobials with respect to control. MIC values are summarized in Table 2 . Tetracycline showed two-folds decrease in MIC $(8 \mu \mathrm{g} / \mathrm{mL}$ to $\leq 4 \mu \mathrm{g} / \mathrm{mL})$ value in $\mathrm{Gr}$. IIA, day 10 , i.e., after biofield treatment in lyophilized state with respect to control (Gr. I). To study the sustained effect of biofield treatment on MIC values, analysis was done after 159 days (Gr. IIB, study I). Results showed that antimicrobials such as cefepime showed two-folds decreased MIC i.e., $16 \mu \mathrm{g} / \mathrm{mL}$ to $\leq 8 \mu \mathrm{g} / \mathrm{mL}$. Beside, altered MIC value was also reported in ESBL-a Scrn $(>4 \mu \mathrm{g} / \mathrm{mL}$ to $\leq 4 \mu \mathrm{g} / \mathrm{mL})$, ESBL-b Scrn $(>1 \mu \mathrm{g} / \mathrm{mL}$ to $\leq 1 \mu \mathrm{g} / \mathrm{mL})$, Cefotaxime $(32 \mu \mathrm{g} / \mathrm{mL}$ to $>32 \mu \mathrm{g} / \mathrm{mL})$, and Norfloxacin $(\leq 4 \mu \mathrm{g} / \mathrm{mL}$ to $8 \mu \mathrm{g} / \mathrm{mL})$ as compared to control (Gr. I).

Gr. IIB, study II, results showed altered MIC values in ten antimicrobials out of thirty-two tested. Decreased value of MIC was reported in case of aztreonam $(>16 \mu \mathrm{g} / \mathrm{mL}$ to $16 \mu \mathrm{g} / \mathrm{mL})$, while two-folds decreased MIC in moxifloxacin $(4 \mu \mathrm{g} / \mathrm{mL}$ to $\leq 2 \mu \mathrm{g} / \mathrm{mL})$, tetracycline $(8 \mu \mathrm{g} / \mathrm{mL}$ to $\leq 4 \mu \mathrm{g} / \mathrm{mL}$ ) on day 10 (Gr. IIB, study II) as compared to control (Gr. I). Cefotaxime showed altered MIC $(32 \mu \mathrm{g} / \mathrm{mL}$ to $>32$ $\mu \mathrm{g} / \mathrm{mL}$ ) on day 5 and 15 , as compared to control (Gr. I). Norfloxacin $(\leq 4 \mu \mathrm{g} / \mathrm{mL}$ to $8 \mu \mathrm{g} / \mathrm{mL})$ showed two-folds decreased MIC on day 5 and 10, as compared to Gr. IIB, study I. Ciprofloxacin, Gatifloxacin and Norfloxacin showed increased MIC values on day 15 (Gr. IIB, study II) compared to control, while MIC value of moxifloxacin and Piperacillin was altered on day 5, and 15 as compared to control. Rest of the antimicrobials did not show any alteration in MIC value, after treatment in any group with respect to control.

\section{Biochemical reaction study}

The biochemical reactions of $P$. fluorescens are reported in Table 3. After biofield treatment, $12.12 \%$ alteration in biochemical reactions was reported. Arginine and cetrimide showed negative reactions (i.e., positive (+) to negative (-)), while glucose showed positive reaction (i.e., negative $(-)$ to positive $(+))$ in Gr. IIA, day 10 as compared to control (Gr. I).

Further, to check the sustained effect on tested biochemicals, analysis was performed on day 159 , and the results showed alteration in cetrimide i.e., again positive reaction on day 159 (Gr. IIB, study I), as compared to results of day 10 (Gr. IIA). However, cetrimide and kanamycin showed negative reaction after retreatment in revived state on day 5, 10, and 15 (Gr. IIB, study II) as compared to control. Arginine showed negative reaction on day 5 , while glucose showed positive reaction on day 5 and 15 as compared to control (Gr. I). Rest of the twenty-nine biochemical did not show any alteration in reaction pattern with respect to control.

\section{Biotype number study}

The biotype number of $P$. fluorescens was determined on MicroScan Walk-Away processed panel, using biochemical reactions data. Biotype numbers with organism identification are summarized in Table 4. The result showed an alteration in biotype number $(4000$ 0043) in Gr. IIA, day 10 and in Gr. IIB, studies II, day 10 with identified organism as Vibrio fluvialis as compared to control. Further results showed an alteration of biotype numbers 4000 0043/0004 0022, with altered species as Vibrio fluvialis/Pseudomonas spp., as compared to control. After retreatment results showed alteration in biotype number on day 5, 10 and 15 in Gr. IIB, study II as compared to control. Biofield treatment showed altered biotype numbers i.e., 4000 0043, 02040622 , and 40002043 observed on day 5, 10, and 15 respectively in Gr. IIB, study II with respect to control. Altered microorganism was found on day 15 as Vibrio fluvialis with respect to control (Gr. I). 
Citation: Trivedi MK, Branton A, Trivedi D, Nayak G, Gangwar M, et al. (2015) Bacterial Identification Using 16S rDNA Gene Sequencing and Antibiogram Analysis on Biofield Treated Pseudomonas fluorescens. Clin Med Biochemistry Open Access 1: 101. doi:10.4172/24712663.1000101

Page 4 of 9

\begin{tabular}{|c|c|c|c|c|c|c|c|}
\hline \multirow[t]{2}{*}{ S No } & \multirow[t]{2}{*}{ Antimicrobial } & \multirow{2}{*}{$\begin{array}{c}\text { Gr. I } \\
\text { Control }\end{array}$} & \multirow{2}{*}{$\begin{array}{l}\text { Gr. IIA } \\
\text { Day } 10\end{array}$} & \multirow{2}{*}{$\begin{array}{c}\text { Gr. IIB, Study I } \\
\text { Day } 159\end{array}$} & \multicolumn{3}{|c|}{ Gr. IIB, Study II } \\
\hline & & & & & $\begin{array}{l}\text { Day } \\
+5\end{array}$ & $\begin{array}{l}\text { Day } \\
+10\end{array}$ & $\begin{array}{l}\text { Day } \\
+15\end{array}$ \\
\hline 1. & Amikacin & $S$ & $S$ & $S$ & $S$ & $S$ & $S$ \\
\hline 2. & Aztreonam & $\mathrm{R}$ & $\mathrm{R}$ & $\mathrm{R}$ & $\mathrm{R}$ & 1 & $\mathrm{R}$ \\
\hline 3. & Cefepime & 1 & 1 & $S$ & $S$ & $S$ & $S$ \\
\hline 4. & Cefotaxime & 1 & I & $\mathrm{R}$ & $\mathrm{R}$ & 1 & $\mathrm{R}$ \\
\hline 5. & Ceftazidime & $S$ & $\mathrm{~S}$ & $S$ & $S$ & $S$ & $S$ \\
\hline 6. & Ceftriaxone & 1 & 1 & 1 & I & 1 & I \\
\hline 7. & Chloramphenicol & $\mathrm{R}$ & $\mathrm{R}$ & $\mathrm{R}$ & $\mathrm{R}$ & $\mathrm{R}$ & $\mathrm{R}$ \\
\hline 8. & Ciprofloxacin & $\mathrm{S}$ & $\mathrm{S}$ & $\mathrm{S}$ & $S$ & $S$ & I \\
\hline 9. & Gentamicin & $S$ & $\mathrm{~S}$ & $S$ & $\mathrm{~S}$ & $\mathrm{~S}$ & $S$ \\
\hline 10. & Imipenem & $S$ & $S$ & $S$ & $S$ & $S$ & $S$ \\
\hline 11. & Levofloxacin & $S$ & $S$ & $S$ & $S$ & $S$ & $S$ \\
\hline 12. & Meropenem & $S$ & $S$ & $S$ & $S$ & $S$ & $S$ \\
\hline 13. & Piperacillin/tazobactam & $S$ & $\mathrm{~S}$ & $S$ & $\mathrm{~S}$ & $S$ & S \\
\hline 14. & Piperacillin & $S$ & $\mathrm{~S}$ & $\mathrm{~S}$ & I & $S$ & I \\
\hline 15. & Tetracycline & 1 & $S$ & 1 & 1 & $S$ & 1 \\
\hline 16. & Ticarcillin/k-clavulanate & $\mathrm{R}$ & $\mathrm{R}$ & $\mathrm{R}$ & $\mathrm{R}$ & $\mathrm{R}$ & $\mathrm{R}$ \\
\hline 17. & Tobramycin & $S$ & $S$ & $S$ & $S$ & $S$ & $\mathrm{~S}$ \\
\hline 18. & Trimethoprim/sulfamethoxazole & $\mathrm{R}$ & $\mathrm{R}$ & $\mathrm{R}$ & $\mathrm{R}$ & $\mathrm{R}$ & $\mathrm{R}$ \\
\hline
\end{tabular}

Table 1: Effect of biofield treatment on antimicrobial susceptibility of Pseudomonas fluorescens. R: Resistant; I: Intermediate; S: Susceptible; Gr.: Group; Antimicrobial susceptibility pattern in control and treated groups were evaluated using automated Micro Scan Walk-Away ${ }^{\circledR}$ system using NBPC30 panel.

\begin{tabular}{|c|c|c|c|c|c|c|c|}
\hline \multirow[t]{2}{*}{ S. No. } & \multirow[t]{2}{*}{ Antimicrobial } & \multirow{2}{*}{$\begin{array}{c}\text { Gr. I } \\
\text { Control }\end{array}$} & \multirow{2}{*}{$\begin{array}{l}\text { Gr. IIA } \\
\text { Day } 10\end{array}$} & \multirow{2}{*}{$\begin{array}{c}\text { Gr. IIB, Study I } \\
\text { Day } 159\end{array}$} & \multicolumn{3}{|c|}{ Gr. IIB, Study II } \\
\hline & & & & & $\begin{array}{c}\text { Day } \\
+5\end{array}$ & $\begin{array}{l}\text { Day } \\
+10\end{array}$ & $\begin{array}{l}\text { Day } \\
+15\end{array}$ \\
\hline 1. & Amikacin & $\leq 16$ & $\leq 16$ & $\leq 16$ & $\leq 16$ & $\leq 16$ & $\leq 16$ \\
\hline 2. & Amoxicillin/k-clavulanate & $>16 / 8$ & $>16 / 8$ & $>16 / 8$ & $>16 / 8$ & $>16 / 8$ & $>16 / 8$ \\
\hline 3. & Ampicillin/sulbactam & $>16 / 8$ & $>16 / 8$ & $>16 / 8$ & $>16 / 8$ & $>16 / 8$ & $>16 / 8$ \\
\hline 4. & Ampicillin & $>16$ & $>16$ & $>16$ & $>16$ & $>16$ & $>16$ \\
\hline 5. & Aztreonam & $>16$ & $>16$ & $>16$ & $>16$ & 16 & $>16$ \\
\hline 6. & Cefazolin & $>16$ & $>16$ & $>16$ & $>16$ & $>16$ & $>16$ \\
\hline 7. & Cefepime & 16 & 16 & $\leq 8$ & $\leq 8$ & $\leq 8$ & $\leq 8$ \\
\hline 8. & Cefotaxime & 32 & 32 & $>32$ & $>32$ & 32 & $>32$ \\
\hline 9. & Cefotetan & $>32$ & $>32$ & $>32$ & $>32$ & $>32$ & $>32$ \\
\hline 10. & Cefoxitin & $>16$ & $>16$ & $>16$ & $>16$ & $>16$ & $>16$ \\
\hline 11. & Ceftazidime & $\leq 8$ & $\leq 8$ & $\leq 8$ & $\leq 8$ & $\leq 8$ & $\leq 8$ \\
\hline 12. & Ceftriaxone & 32 & 32 & 32 & 32 & 32 & 32 \\
\hline 13. & Cefuroxime & $>16$ & $>16$ & $>16$ & $>16$ & $>16$ & $>16$ \\
\hline 14. & Cephalothin & $>16$ & $>16$ & $>16$ & $>16$ & $>16$ & $>16$ \\
\hline 15. & Chloramphenicol & $>16$ & $>16$ & $>16$ & $>16$ & $>16$ & $>16$ \\
\hline 16. & Ciprofloxacin & $\leq 1$ & $\leq 1$ & $\leq 1$ & $\leq 1$ & $\leq 1$ & 2 \\
\hline 17. & ESBL-a Scrn & $>4$ & $>4$ & $\leq 4$ & $>4$ & $>4$ & $>4$ \\
\hline
\end{tabular}


Citation: Trivedi MK, Branton A, Trivedi D, Nayak G, Gangwar M, et al. (2015) Bacterial Identification Using 16S rDNA Gene Sequencing and Antibiogram Analysis on Biofield Treated Pseudomonas fluorescens. Clin Med Biochemistry Open Access 1: 101. doi:10.4172/24712663.1000101

Page 5 of 9

\begin{tabular}{|c|c|c|c|c|c|c|c|}
\hline 18. & ESBL-b Scrn & $>1$ & $>1$ & $\leq 1$ & $>1$ & $>1$ & $>1$ \\
\hline 19. & Gatifloxacin & $\leq 2$ & $\leq 2$ & $\leq 2$ & $\leq 2$ & $\leq 2$ & 4 \\
\hline 20. & Gentamicin & $\leq 4$ & $\leq 4$ & $\leq 4$ & $\leq 4$ & $\leq 4$ & $\leq 4$ \\
\hline 21. & Imipenem & $\leq 4$ & $\leq 4$ & $\leq 4$ & $\leq 4$ & $\leq 4$ & $\leq 4$ \\
\hline 22. & Levofloxacin & $\leq 2$ & $\leq 2$ & $\leq 2$ & $\leq 2$ & $\leq 2$ & $\leq 2$ \\
\hline 23. & Meropenem & $\leq 4$ & $\leq 4$ & $\leq 4$ & $\leq 4$ & $\leq 4$ & $\leq 4$ \\
\hline 24. & Moxifloxacin & 4 & 4 & 4 & $>4$ & $\leq 2$ & $>4$ \\
\hline 25. & Nitrofurantoin & $>64$ & $>64$ & $>64$ & $>64$ & $>64$ & $>64$ \\
\hline 26. & Norfloxacin & $\leq 4$ & $\leq 4$ & 8 & $\leq 4$ & $\leq 4$ & 8 \\
\hline 27. & Piperacillin/tazobactam & $\leq 16$ & $\leq 16$ & $\leq 16$ & $\leq 16$ & $\leq 16$ & $\leq 16$ \\
\hline 28. & Piperacillin & $\leq 16$ & $\leq 16$ & $\leq 16$ & 64 & $\leq 16$ & 64 \\
\hline 29. & Tetracycline & 8 & $\leq 4$ & 8 & 8 & $\leq 4$ & 8 \\
\hline 30. & Ticarcillin/k-clavulanate & $>64$ & $>64$ & $>64$ & $>64$ & $>64$ & $>64$ \\
\hline 31. & Tobramycin & $\leq 4$ & $\leq 4$ & $\leq 4$ & $\leq 4$ & $\leq 4$ & $\leq 4$ \\
\hline 32. & Trimethoprim/ Sulfamethoxazole & $>2 / 38$ & $>2 / 38$ & $>2 / 38$ & $>2 / 38$ & $>2 / 38$ & $>2 / 38$ \\
\hline
\end{tabular}

Table 2: Minimum inhibitory concentration (MIC) of tested antimicrobials against Pseudomonas fluorescens. MIC values are presented in $\mu \mathrm{g} / \mathrm{mL}$; Gr.: Group; ESBL-a, b Scrn: Extended spectrum beta-lactamase a, b Screen; MIC values in control and treated groups were evaluated using automated Micro Scan Walk-Away ${ }^{\circledR}$ system using NBPC30 panel.

\begin{tabular}{|c|c|c|c|c|c|c|c|c|}
\hline \multirow{3}{*}{ S. No. } & \multirow{3}{*}{ Code } & \multirow{3}{*}{ Biochemical } & \multicolumn{6}{|c|}{ Type of Response } \\
\hline & & & \multirow{2}{*}{$\begin{array}{c}\text { Gr. I } \\
\text { Control }\end{array}$} & \multirow{2}{*}{$\begin{array}{l}\text { Gr. IIA } \\
\text { Day } 10\end{array}$} & \multirow{2}{*}{$\begin{array}{c}\text { Gr.IIB, } \\
\text { Study I } \\
\text { Day } 159\end{array}$} & \multicolumn{3}{|c|}{ Gr. IIB, Study II } \\
\hline & & & & & & $\begin{array}{l}\text { Day } \\
+5\end{array}$ & $\begin{array}{l}\text { Day } \\
+10\end{array}$ & $\begin{array}{l}\text { Day } \\
+15\end{array}$ \\
\hline 1. & ACE & Acetamide & - & - & - & - & - & - \\
\hline 2. & ADO & Adonitol & - & - & - & - & - & - \\
\hline 3. & ARA & Arabinose & - & - & - & - & - & - \\
\hline 4. & ARG & Arginine & + & - & - & - & + & + \\
\hline 5. & CET & Cetrimide & + & - & + & - & - & - \\
\hline 6. & CF8 & Cephalothin & + & + & + & + & + & + \\
\hline 7. & CIT & Citrate & + & + & + & + & + & + \\
\hline 8. & CL4 & Colistin & - & - & - & - & - & - \\
\hline 9. & ESC & Esculin hydrolysis & - & - & - & - & - & - \\
\hline 10. & FD64 & Nitrofurantoin & + & + & + & + & + & + \\
\hline 11. & GLU & Glucose & - & + & + & + & - & + \\
\hline 12. & $\mathrm{H} 2 \mathrm{~S}$ & Hydrogen sulfide & - & - & - & - & - & - \\
\hline 13. & IND & Indole & - & - & - & - & - & - \\
\hline 14. & INO & Inositol & - & - & - & - & - & - \\
\hline 15. & $\mathrm{~K} 4$ & Kanamycin & + & + & + & - & - & - \\
\hline 16. & LYS & Lysine & - & - & - & - & - & - \\
\hline 17. & MAL & Malonate & - & - & - & - & - & - \\
\hline 18. & MEL & Melibiose & - & - & - & - & - & - \\
\hline 19. & NIT & Nitrate & - & - & - & - & - & - \\
\hline 20. & OF/G & $\begin{array}{l}\text { Oxidation-fermentation/ } \\
\text { glucose }\end{array}$ & + & + & + & + & + & + \\
\hline 21. & ONPG & Galactosidase & - & - & - & - & - & - \\
\hline 22. & ORN & Ornithine & - & - & - & - & - & - \\
\hline 23. & OXI & Oxidase & + & + & + & + & + & + \\
\hline
\end{tabular}


Citation: Trivedi MK, Branton A, Trivedi D, Nayak G, Gangwar M, et al. (2015) Bacterial Identification Using 16S rDNA Gene Sequencing and Antibiogram Analysis on Biofield Treated Pseudomonas fluorescens. Clin Med Biochemistry Open Access 1: 101. doi:10.4172/24712663.1000101

Page 6 of 9

\begin{tabular}{|c|c|c|c|c|c|c|}
\hline 24. & P4 & Penicillin & + & + & + \\
\hline 25. & RAF & Raffinose & - & - & - \\
\hline 26. & RHA & Rhamnose & - & - & - & - \\
\hline 27. & SOR & Sorbitol & - & - & - \\
\hline 28. & SUC & Sucrose & - & - & - \\
\hline 29. & TAR & Tartrate & - & - & - \\
\hline 30. & TDA & Tryptophan deaminase & - & - & - \\
\hline 31. & TO4 & Tobramycin & - & - & - \\
\hline 32. & URE & Urea & Voges-Proskauer & - & - & - \\
\hline 33. & VP & - & - & - \\
\hline
\end{tabular}

Table 3: Effect of biofield treatment on biochemical reactions of Pseudomonas fluorescens. -: negative; +: positive; Gr.: Group; Biochemical reactions in control and treated groups were evaluated using automated MicroScan Walk-Away ${ }^{\circledR}$ system using NBPC30 panel.

\begin{tabular}{|c|c|c|c|c|c|c|}
\hline \multirow[t]{2}{*}{ Feature } & \multirow{2}{*}{$\begin{array}{c}\text { Gr. I } \\
\text { Control }\end{array}$} & \multirow{2}{*}{$\begin{array}{l}\text { Gr. IIA } \\
\text { Day } 10\end{array}$} & \multirow{2}{*}{$\begin{array}{l}\text { Gr. IIB, } \\
\text { Study I } \\
\text { Day } 159\end{array}$} & \multicolumn{3}{|c|}{ Gr. IIB, Study II } \\
\hline & & & & $\begin{array}{l}\text { Day } \\
+5\end{array}$ & $\begin{array}{l}\text { Day } \\
+10\end{array}$ & $\begin{array}{l}\text { Day } \\
+15\end{array}$ \\
\hline Biotype number & 02041722 & 40000043 & $\begin{array}{l}40000043 / \\
00040022\end{array}$ & 40000043 & 02040622 & 40002043 \\
\hline Organism identification & P. fluorescens & Vibrio fluvialis & $\begin{array}{c}\text { Vibrio fluvialis/ } \\
\text { Pseudomonas spp. }\end{array}$ & Vibrio fluvialis & P. fluorescens/putida & Vibrio fluvialis \\
\hline
\end{tabular}

Table 4: Effect of biofield treatment on biotype number of Pseudomonas fluorescens. Biotype numbers and organism identification in control and treated groups were evaluated using automated MicroScan Walk-Away ${ }^{\circledR}$ system using NBPC30 panel.

\section{Amplification and sequence determination by $16 \mathrm{~S}$ rDNA}

In order to confirm the PCR-based identification results, $16 \mathrm{~S}$ rDNA sequence analysis was performed in biofield treated lyophilized $P$. fluorescens strain. The alignment and assessment of the gene sequences data were performed by comparing with the sequences available in GenBank database of NCBI, using the algorithm BLASTn program. The phylogenetic tree was constituted using BLAST-Webpage (NCBI). Based on nucleotides homology and phylogenetic analysis the Microbe (Sample 2A) was detected to be Pseudomonas entomophila (GenBank Accession Number: AY907566) with 96\% identity of gene sequencing data. Ten closely related bacterial species and P. fluorescens were considered as Operational Taxonomic Units (OTUs) in order to investigate the phylogenetic relationship of $P$. fluorescens among other ten related species (Figure 1). Total 1482 base nucleotide of $16 \mathrm{~S}$ rDNA gene sequences were analyzed by multiple alignments using ClustalW of MEGA3.1 program [24]. Based on the phylogenetic tree and 16S rDNA sequencing, the nearest homolog genus-species was found to be Pseudomonas fluorescens (Accession No. EF672049). Other closely related homologs of $P$. fluorescens can be found from the sequence alignment as shown in Table 5. Distance matrix between the 16S-rDNA sequences of 11 pathogens was analyzed based on nucleotide sequence homology using Kimura-2 Parameter. According to the data in Table 6 , the lowest value of genetic distance from sample $2 \mathrm{~A}$ was 0.003 base substitutions per site. Total 11 sequences of base substitutions per site from pairwise distance analysis were shown in Table 6.

\section{Discussion}

P. fluorescens causes bacteremia and pseudo bacteremia in immunocompromised patients $[25,26]$. Discovering a new drug chemical moiety against resistant strain will require huge effort and time, and unfortunately, new drugs have been accompanied by the

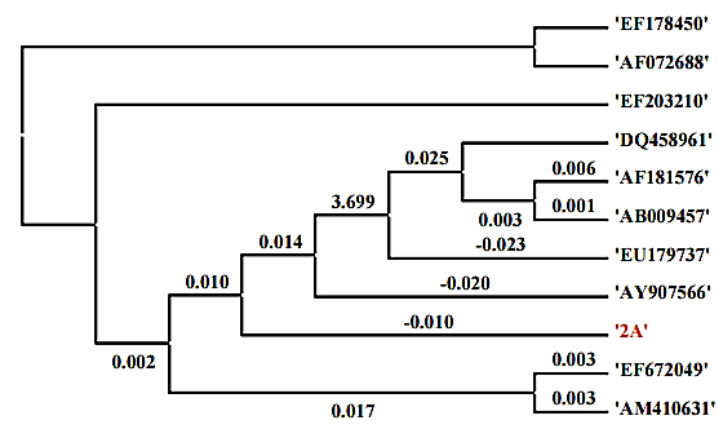

Figure 1: Phylogenetic tree based on partial sequence analysis of $16 \mathrm{~S}$ rDNA gene sequencing showing the cluster of closely related species using MEGA 3.1 software by Neighbor joining method. Numbers represent GenBank accession numbers.

quick emergence of resistant via various natural mechanisms. However, biofield treatment on microbes has been reported as an alternate approach to improve the susceptibility pattern of antimicrobial up to a great extent.

In the preset work, authors investigated the effect of biofield treatment on lyophilized strain of $P$. fluorescens and its sustained effect was studied at day 159 , followed by retreatment. The results showed alteration in antimicrobial sensitivity in case of aztreonam, cefepime, and tetracycline in different assessment days. P. fluorescens was highly sensitive to kanamycin, tetracycline, and ciprofloxacin at very low concentration [27]. According to Benito et al. P. fluorescens is susceptible to both gentamicin and Ceftazidime [28]. Gentamycin and Ceftazidime are the choice of drug used against $P$. fluorescens infection. Experimental control results were well supported with literature data [29]. According to Greenberg et al. Amikacin with aztreonam has synergistic effect against Pseudomonas aeruginosa and other Gram- 
Citation: Trivedi MK, Branton A, Trivedi D, Nayak G, Gangwar M, et al. (2015) Bacterial Identification Using 16S rDNA Gene Sequencing and Antibiogram Analysis on Biofield Treated Pseudomonas fluorescens. Clin Med Biochemistry Open Access 1: 101. doi:10.4172/24712663.1000101

Page 7 of 9

\begin{tabular}{|c|c|c|c|}
\hline Alignment View & AN & Alignment results & Sequence description \\
\hline \begin{tabular}{|l|l} 
\\
\end{tabular} & $2 \mathrm{~A}$ & 0.99 & Sample studied \\
\hline \begin{tabular}{|l|} 
\\
\end{tabular} & AF072688 & 1.00 & Pseudomonas mosselii strain CIP 105259 \\
\hline \begin{tabular}{|l|l}
-1 \\
\end{tabular} & AF181576 & 0.98 & Pseudomonas monteilii \\
\hline \begin{tabular}{|l|l} 
\\
\end{tabular} & EF203210 & 0.97 & Pseudomonas putida strain J312 \\
\hline \begin{tabular}{|l|l|} 
\\
\end{tabular} & EU179737 & 0.98 & Pseudomonas putida strain MG-Y2 \\
\hline \begin{tabular}{|l|l|} 
\\
\end{tabular} & AY907566 & 0.96 & Pseudomonas entomophila strain L48 \\
\hline \begin{tabular}{|c|c|} 
\\
\end{tabular} & DQ458961 & 0.99 & Pseudomonas putida \\
\hline \begin{tabular}{|c|} 
\\
\end{tabular} & AB009457 & 1.00 & Pseudomonas plecoglossicida \\
\hline$\square$ & EF178450 & 0.98 & Pseudomonas entomophila strain $2 \mathrm{P} 25$ \\
\hline$\square$ & EF672049 & 0.96 & Pseudomonas fluorescens strain Mc07 \\
\hline \begin{tabular}{|l|l} 
\\
\end{tabular} & AM410631 & 0.97 & Pseudomonas fluorescens strain 9zhy \\
\hline
\end{tabular}

Table 5: The closest sequences of Pseudomonas fluorescens from sequence alignment using NCBI GenBank and ribosomal database project (RDP). AN: GenBank Accession Number. Alignment results and sequence description has been obtained from the blast results of GenBank database of National Center for Biotechnology Information (NCBI) using the algorithm BLASTn program.

\begin{tabular}{|c|c|c|c|c|c|c|c|c|c|c|c|c|}
\hline \multicolumn{13}{|c|}{ Distance Matrix } \\
\hline AN & & 1 & 2 & 3 & 4 & 5 & 6 & 7 & 8 & 9 & 10 & 11 \\
\hline EF203210 & 1 & - & -2.755 & -2.766 & -2.760 & -2.708 & 1 & 1 & 0.978 & 0.978 & 0.999 & 0.999 \\
\hline DQ458961 & 2 & 3.755 & - & 0.991 & 0.996 & 0.996 & -2.755 & -2.755 & -2.767 & -2.767 & -2.703 & -2.708 \\
\hline AF181576 & 3 & 3.766 & 0.009 & - & 0.992 & 0.992 & -2.766 & -2.766 & -2.778 & -2.778 & -2.713 & -2.719 \\
\hline AB009457 & 4 & 3.760 & 0.004 & 0.008 & - & 0.995 & -2.760 & -2.760 & -2.772 & -2.772 & -2.708 & -2.713 \\
\hline EU179737 & 5 & 3.708 & 0.004 & 0.008 & 0.005 & - & -2.708 & -2.708 & -2.717 & -2.717 & -2.657 & -2.662 \\
\hline EF178450 & 6 & 0.000 & 3.755 & 3.766 & 3.760 & 3.708 & - & 1 & 0.978 & 0.978 & 0.999 & 0.999 \\
\hline AF072688 & 7 & 0.000 & 3.755 & 3.766 & 3.760 & 3.708 & 0.000 & - & 0.978 & 0.978 & 0.999 & 0.999 \\
\hline EF672049 & 8 & 0.022 & 3.767 & 3.778 & 3.772 & 3.717 & 0.022 & 0.022 & - & 0.994 & 0.979 & 0.977 \\
\hline AM410631 & 9 & 0.022 & 3.767 & 3.778 & 3.772 & 3.717 & 0.022 & 0.022 & 0.006 & - & 0.979 & 0.977 \\
\hline AY907566 & 10 & 0.001 & 3.703 & 3.713 & 3.708 & 3.657 & 0.001 & 0.001 & 0.021 & 0.021 & - & 0.997 \\
\hline $2 \mathrm{~A}$ & 11 & 0.001 & 3.708 & 3.719 & 3.713 & 3.662 & 0.001 & 0.001 & 0.024 & 0.024 & 0.003 & - \\
\hline
\end{tabular}

Table 6: Distance matrix between the 16S rDNA sequences of 11 pathogens based on nucleotide sequence homology (Using Kimura-2 Parameter) of $P$ seudomonas fluorescens. AN: GenBank Accession Number. Total 1482 base nucleotide of $16 \mathrm{~S}$ rDNA gene sequences were analyzed by multiple alignments using ClustalW program. Pairwise distance (lower left) and number of nucleotide difference (upper-right) for $16 \mathrm{~S}$ forward and reverse primer was presented using Kimura-2 Parameters.

negative organisms [30]. Although, P. fluorescens is susceptible in all the groups against Amikacin before and after treatment, but found resistant against aztreonam in control (Gr. I). After biofield treatment in lyophilized as well as retreatment in revived state showed improved sensitivity pattern of aztreonam from resistant to intermediate. Also, MIC results were well supported (i.e., decreased MIC to $16 \mu \mathrm{g} / \mathrm{mL}$ ) with improved sensitivity data as compared to control. Likewise, cefepime efficacy for the treatment of pneumonia in hospitalized patients against Pseudomonas infections was well reported [31,32]. The experimental results showed improve efficacy of cefepime in terms of sensitivity as well as MIC value (two-folds decrease) after biofield retreatment as compared to control. Biofield treatment on Pseudomonas might up-regulated the efflux membrane, or alter the function of inducible AmpC $\beta$-lactamase enzymes, which could results in altered sensitivity. Similarly, use of tetracycline against Pseudomonas infections has been well studied [33]. Biofield treatment showed improved sensitivity as well as decreased MIC value of tetracycline. Moxifloxacin and extended spectrum beta lactamases (ESBL) showed improved susceptibility against $P$. fluorescens with respect to control results.

Characteristic biochemical reactions of Pseudomonas species showed negative reaction in Voges-Proskauer, indole, and methyl red, hydrogen sulfide, glucose while positive reaction in catalase test. While some species i.e., P.fluorescens showed a positive oxidase test [34]. In this study, results of biochemical reactions were showed positive reaction in arginine, cetrimide, kanamycin while negative reaction in VogesProskauer, arabinose, colistin, rhaminose, malonate, melibiose, nitrate, galactosidase, ornithine, raffinose, sorbitol, sucrose, and tobramycin. After biofield treatment, results showed altered biochemical reactions of arginine, cetrimide, glucose, and kanamycin. Study using different clinical isolates of Pseudomonas spp. showed that P. fluorescens did not have the capacity to grow or utilize glucose in media, proof to be a real feature of pseudomonads [34]. Biofield treatment might cause some changes at enzymatic or metabolic pathway leading to significant phenotypic alteration in $P$. fluorescens, which may results in utilizing glucose in growth media. Altered biochemical reactions were results in identification of changed biotype number and species. Biotyping makes use of the pattern of metabolic activities expressed by an isolate, colonial morphology and environmental tolerances. In this experiment, biotyping was performed using automated system, results showed significant change in the biotype number with identification of new species in treated groups, and organism identified as Vibrio fluvialis with respect to control. Additionally, molecular methods were further studied to study and confirm the changes in species using $16 \mathrm{~S}$ rDNA sequencing method.

Genotypic identification methods would be expected to circumvent this change in species as evidenced by altered biochemical reactions and 
Citation: Trivedi MK, Branton A, Trivedi D, Nayak G, Gangwar M, et al. (2015) Bacterial Identification Using 16S rDNA Gene Sequencing and Antibiogram Analysis on Biofield Treated Pseudomonas fluorescens. Clin Med Biochemistry Open Access 1: 101. doi:10.4172/24712663.1000101

Page 8 of 9

biotype number. Molecular assays based on $16 \mathrm{~S}$ rDNA amplification protocol have been described. These include PCR assays and DNA amplification using standard forward and reverse $16 \mathrm{~S}$ universal primers. 16S rDNA amplification protocol has been commonly used as a taxonomic "gold standard" in identification and determining the phylogenies of bacterial species [35]. Selective amplification using $16 \mathrm{~S}$ rDNA was well reported to detect and differentiate Pseudomonas species from clinical and environmental samples [36]. Based on the BLASTn analysis, the sample $2 \mathrm{~A}$ was identified as Pseudomonas entomophila with $96 \%$ similarity in gene sequence. The phylogenetic tree diagram (Figure 1) showed that nearest homolog species was found to be Pseudomonas fluorescens. Above results suggest that biofield treatment has significant impact on Pseudomonas species, which was well supported with $16 \mathrm{~S}$ rDNA analysis.

Thus, above results indicates that biofield treatment in lyophilized as well as revived state showed significant results in terms of improved antimicrobials sensitivity, decreased MIC, altered characteristic biochemical reactions followed by change in biotype number. Study results conclude that biofield treatment has significant and sustained effect at phenotypic level for a total duration of 174 days. Study design and results showed that alterations might occur even after storage of sample in $-70^{\circ} \mathrm{C}$ for 159 days. This suggests that Mr. Trivedi's unique biofield treatment has the ability to alter the antimicrobial sensitivity in treated pathogenic microbes even in the lyophilized storage condition for long duration. Bioelectromagnetic-based therapies and biofield therapies are energy therapies that use or manipulate the energy fields to promote health and healing. These energy therapies are well described under energy medicine by National Center for Complementary and Alternative Medicine (NIH/NCCAM). Biofield therapies are very popular in biomedical heath care systems [37]. Biofield healing treatment on pathogenic strains might involve change in cell receptor protein due to electromagnetic field. Healing therapy or therapeutic touch might modify ligand-receptor interaction, which causes alteration in phenotypic characteristics [38]. Hence, it is assumed that biofield treatment made some alteration at enzymatic or genetic level, which manifested in phenotypic alteration.

\section{Conclusions}

Improved antimicrobial sensitivity and decreased MIC value of aztreonam, cefepime, moxifloxacin, and tetracycline in biofield treated Pseudomonas fluorescens showed significant impact of Mr. Trivedi's biofield energy treatment. This approach can be used as a treatment approach in complementary and alternate medicine. Significant alteration in antimicrobial data was well supported with altered biochemical reactions along with biotype number. Molecular approach using standard $16 \mathrm{~S}$ rDNA analysis showed that biofield treatment has significant impact on $P$. fluorescens, and sample identified as Pseudomonas entomophila with $96 \%$ identity using gene sequencing data. However, the closest homolog species was detected to be Pseudomonas fluorescens. Based on these results, it seems that $\mathrm{Mr}$. Trivedi's biofield energy treatment could be used as better alternate of existing drug therapy in future.

\section{Acknowledgements}

Authors gratefully acknowledged the whole team of PD Hinduja National Hospital and MRC, Mumbai, Microbiology Lab for conduction the study and also thankful to Trivedi Science ${ }^{T \omega}$, Trivedi Master Wellness ${ }^{\text {Tw }}$ and Trivedi Testimonials for their generous support in experimental works.

\section{References}

1. Verbist L (1993) Epidemiology and sensitivity of 8625 ICU and hematology/ oncology bacterial isolates in Europe. International Study Group. Scand. J Infect Dis 91: 14-24.

2. Mandell GL, Bennett JE, Dolin R (2004) Pseudomonas aeruginosa. In Mandell Douglas, and Bennett's principles and practice of infectious diseases. (6thedn), Churchill Livingstone, New York, US.

3. Hsueh PR, Teng LJ, Pan HJ, Chen YC, Sun CC, et al. (1998) Outbreak of Pseudomonas fluorescens bacteremia among oncology patients. J Clin Microbiol 36: 2914-2917.

4. Palleroni NJ (1991) Present situation of the taxonomy of aerobic Pseudomonas. In: Pseudomonas molecular biology and biotechnology. American Society for Microbiology, Washington, DC.

5. Paulsen IT, Press CM, Ravel J, Kobayashi DY, Myers GS, et al. (2005) Complete genome sequence of the plant commensal Pseudomonas fluorescens Pf-5. Nat Biotechnol 23: 873-878.

6. http://www.ncbi.nlm.nih.gov/entrez/query.fcgi?db=genomeprj\&cmd=Retrieve\& dopt=Overview\&list_uids $=12300$.

7. Smith AU (1954) Effect of low temperature on living cells and tissues. Biological applications of freezing and drying. Academic Press, Inc., New York.

8. Mazur PM, Rhian MA, Mahlandt BG (1957) Survival of Pasteurella tularensis in sugar solutions after cooling and warming at sub-zero temperatures. J Bacteriol 73: 394-397.

9. Harris RJC (1954) The preservation of bacteria. Biological applications of freezing and drying. Academic Press, Inc., New York.

10. Heckly RJ (1961) Preservation of bacteria by lyophilization. Adv Appl Microbiol 3: 1-76.

11. Dhabade VV, Tallapragada RM, Trivedi MK (2009) Effect of external energy on atomic, crystalline and powder characteristics of antimony and bismuth powders. Bull Mater Sci 32: 471-479.

12. Trivedi MK, Patil S, Tallapragada RM (2013) Effect of biofield treatment on the physical and thermal characteristics of silicon, tin and lead powders. J Material Sci Eng 2: 125.

13. Trivedi MK, Nayak G, Patil S, Tallapragada RM, Latiyal O (2015) Studies of the atomic and crystalline characteristics of ceramic oxide nano powders after bio field treatment. Ind Eng Manage 4: 161

14. Shinde V, Sances F, Patil S, Spence A (2012) Impact of biofield treatment on growth and yield of lettuce and tomato. Aust J Basic Appl Sci 6: 100-105.

15. Sances F, Flora E, Patil S, Spence A, Shinde V (2013) Impact of biofield treatment on ginseng and organic blueberry yield. Agrivita J Agric Sci 35: 2229.

16. Lenssen AW (2013) Biofield and fungicide seed treatment influences on soybean productivity, seed quality and weed community. Agricultural Journal 8: 138-143.

17. Nayak G, Altekar N (2015) Effect of biofield treatment on plant growth and adaptation. J Environ Health Sci 1: 1-9.

18. Trivedi MK, Patil S, Shettigar H, Gangwar M, Jana S (2015) An effect of biofield treatment on multidrug-resistant Burkholderia cepacia: A multihost pathogen. J Trop Dis 3: 167.

19. Trivedi MK, Bhardwaj Y, Patil S, Shettigar H, Bulbule A (2009) Impact of an external energy on Enterococcus faecalis [ATCC-51299] in relation to antimicrobials susceptibility and biochemical reactions-an experimental study. J Accord Integr Med 5: 119-130.

20. Trivedi MK, Patil S, Shettigar H, Bairwa K, Jana S (2015) Phenotypic and biotypic characterization of Klebsiella oxytoca: An impact of biofield treatment J Microb Biochem Technol 7: 203-206.

21. Trivedi MK, Patil S, Shettigar H, Mondal SC, Jana S (2015) Evaluation of biofield modality on viral load of Hepatitis B and C viruses. J Antivir Antiretrovir 7: 083-088.

22. Fader RC, Weaver E, Fossett R, Toyras M, Vanderlaan J, et al. (2013) Multilaboratory study of the biomic automated well-reading instrument versus MicroScan WalkAway for reading MicroScan antimicrobial susceptibility and identification panels. J Clin Microbiol 51: 1548-1554.

23. Alm EW, Oerther DB, Larsen N, Sthal DA, Raskin L (1996) The oligonucleotide probe database. Appl Environ Microbiol 62: 3557-3559. 
Citation: Trivedi MK, Branton A, Trivedi D, Nayak G, Gangwar M, et al. (2015) Bacterial Identification Using 16S rDNA Gene Sequencing and Antibiogram Analysis on Biofield Treated Pseudomonas fluorescens. Clin Med Biochemistry Open Access 1: 101. doi:10.4172/24712663.1000101

Page 9 of 9

24. Kumar S, Tamura K, Nei M (2004) MEGA3: Integrated software for molecular evolutionary genetics analysis and sequence alignment. Brief Bioinform 5: 150163.

25. Donnarumma G, Buommino E, Fusco A, Paoletti I, Auricchio L, et al. (2010) Effect of temperature on the shift of Pseudomonas fluorescens from an environmental microorganism to a potential human pathogen. Int $J$ Immunopathol Pharmacol 23: 227-234.

26. Wong V, Levi K, Baddal B, Turton J, Boswell TC (2011) Spread of Pseudomonas fluorescens due to contaminated drinking water in a bone marrow transplant unit. J Clin Microbiol 49: 2093-2096.

27. Morgan AE (2014) The synergistic effect of gentamicin and ceftazidime against Pseudomonas fluorescens. Bioscience Horizons 7: hzu007.

28. Benito N, Mirelis B, Luz Galvez ML, Vila M, Lopez-Contreras J, et al. (2012) Outbreak of Pseudomonas fluorescens bloodstream infection in a coronary care unit. J Hosp Infect 82: 286-289.

29. Mikura S, Wada H, Okazaki M, Nakamura M, Honda K, et al. (2011) Risk factors for bacteraemia attributable to Pseudomonas aeruginosa resistant to imipenem, levofloxacin, or gentamicin. J Hosp Infect 79: 267-268.

30. Greenberg RN, Bollinger M, Compton J (1986) Synergistic activity of amikacin with aztreonam against Pseudomonas aeruginosa and other gram-negative organisms. Clin Ther 8: 354-358.

31. Zervos M, Nelson M, Bernstein J, Kernodle D, McCabe R, et al. (1998) Cefepime versus ceftriaxone for empiric treatment of hospitalized patients with community-acquired pneumonia. Antimicrob Agents Chemother 42: 729-733.
32. Stes P, Goossens H (1996) Cefepime activity against Pseudomonas aeruginosa: Evaluation of Etest and two disc diffusion methods. J Antimicrob Chemother 38: 707-711.

33. O'Brien W, Fyfe C, Grossman T, Chen C, Clark R, et al. (2012) In vitro potency of novel tetracyclines against Pseudomonas aeruginosa and other major Gram-negative pathogens. 22nd European Congress of Clinical Microbiology and Infectious Disease (ECCMID) in London, UK.

34. Rhodes ME (1959) The characterization of Pseudomonas fluorescens. J Gen Microbiol 21: 221-265.

35. Woese CR (1987) Bacterial evolution. Microbiol Rev 51: 221-271.

36. Widmer F, Seidler RJ, Gillevet PM, Watrud LS, Di Giovanni GD (1998) A highly selective PCR protocol for detecting 16S rRNA genes of the genus Pseudomonas (sensu stricto) in environmental samples. Appl Environ Microbiol 64: 2545-2553.

37. Clarke TC, Black LI, Stussman BJ, Barnes PM, Nahin RL (2015) Trends in the use of complementary health approaches among adults: Unites States, 2002 2012. National health statistics reports; No 79. Hyattsville, MD: National Center for Health Statistics.

38. Hintz KJ, Yount GL, Kadar I, Schwartz G, Hammerschlag R, et al. (2003) Bioenergy definitions and research guidelines. Altern Ther Health Med 9: 1330.
OMICS International: Publication Benefits \& Features Unique features:

Increased global visibility of articles through worldwide distribution and indexing Showcasing recent research output in a timely and updated manner

Special issues on the current trends of scientific research

Special features:

700 Open Access Journals

50,000 editorial team

Rapid review process

Quality and quick editorial, review and publication processing

Indexing at PubMed (partial), Scopus, EBSCO, Index Copernicus, Google Scholar etc.

Sharing Option: Social Networking Enabled

Authors, Reviewers and Editors rewarded with online Scientific Credits

Better discount for your subsequent articles

Submit your manuscript at: http://www.omicsonline.org/submission 